\title{
Donepezil, Therapeutic Acetylcholinesterase Inhibitor, Prevents the Progression of Ventricular Dysfunction by Promoting Myocardial Glucose Utilization in Rat Model of Chronic Heart Failure Following Myocardial
} Infarction

\author{
Mikihiko Arikawa $^{1^{*}}$, Yoshihiko Kakinuma ${ }^{2}$, Tatsuya Noguchi ${ }^{1,3}$ and Takayuki Sato ${ }^{1}$ \\ ${ }^{1}$ Department of Cardiovascular Control, Kochi Medical School, Nankoku, Kochi, Japan \\ ${ }^{2}$ Department of Physiology, Nippon Medical School Graduate School of Medicine, Tokyo, Japan \\ ${ }^{3}$ Department of Medical and Geriatrics, Kochi Medical School, Nankoku, Kochi, Japan
}

\begin{abstract}
Background: Donepezil, an acetylcholinesterase inhibitor, is reported to prevent cardiac pumping dysfunction in rats with chronic heart failure (CHF). Because energy substrate switching is a potential therapeutic target for the pharmacological treatment of $\mathrm{CHF}$, we investigated the effect of donepezil on cardiac energy metabolism.

Methods and Results: After induction of myocardial infarction (MI), rats were assigned to untreated and donepezil-treated (DPZ) groups. At the chronic phase of MI, resting heart rate was comparable between the untreated and DPZ groups. Nevertheless, left ventricular contractility evaluated in Langendorff-perfused hearts was significantly improved by donepezil treatment. At the same time point, the expression level of cardiac glucose transporter (GLUT) was significantly higher in the DPZ group than in the untreated group. In vitro studies showed that cultured cardiomyocytes treated with donepezil expressed high levels of GLUT and also exhibited accelerated cellular glucose uptake. Moreover, the beating rate of DPZ cardiomyocytes was approximately 2 -fold higher than that of untreated control cells. Fasentin, a glucose transporter inhibitor, nullified the effects of donepezil, indicating that donepezil increases cellular activity by promoting glucose utilization in cardiomyocytes.
\end{abstract}

Conclusion: The present study suggests that donepezil exhibits cardioprotective action by chronically modulating glucose metabolism in the failing heart.

Keywords: Glucose transporter; Pressure-volume relation; Metabolic remodeling; Substrate preference

\section{Introduction}

The heart has been frequently described as a "metabolic omnivore" because it utilizes a variety of oxidizable substrates such as fatty acids and carbohydrates to produce energy for contraction, either simultaneously or vicariously. Moreover, under stress conditions, the heart readily changes the substrate to the most advantageous one according to the underlying physiological condition $[1,2]$. This metabolic remodeling, so-called substrate switching, occurs in the failing heart, which is characterized by a reduction in fatty acid oxidation and an increase in glucose utilization [3,4]. Although it is still not completely understood whether metabolic remodeling is beneficial or detrimental, stimulating glucose utilization is preferable for the failing heart rather than normalizing substrate metabolism by stimulating fatty acid utilization [5]. Therefore, cardiac metabolism is a potential therapeutic target for the pharmacological treatment of chronic heart failure (CHF), and a drug that has the ability to facilitate glucose utilization can be a novel candidate for the prevention and treatment of CHF.

A series of animal and clinical studies conducted by our group has shown a beneficial effect of donepezil in CHF [6-13]. Although donepezil is widely used as an acetylcholinesterase inhibitor for the treatment of Alzheimer's disease [14,15], our experimental studies have demonstrated that donepezil elicits various effects on the cardiovascular system, independent of cholinesterase inhibitory actions $[10,12,13]$. Without slowing heart rate, donepezil improved long-term survival in animals with CHF through upregulation of anti-apoptotic and angiogenic factors in cardiac and endothelial cells $[12,13]$. However, it remains to be elucidated whether donepezil affects metabolic remodeling in CHF.
In the present study, we focused on donepezil-induced alterations in cardiac expression of genes/proteins related to energy metabolism in an ischemic CHF model and in cultured rat cardiomyocytes. The present results show that chronic oral administration of donepezil prevents the progression of left ventricular dysfunction after myocardial infarction (MI) without affecting heart rate, and that donepezil increases cellular glucose utilization by upregulating the expression of glucose transporters (GLUTs). Here we suggest that donepezilinduced acceleration of myocardial glucose utilization may represent a mechanism for cardioprotection in the failing heart.

\section{Materials and Methods}

\section{Animal}

The care and use of laboratory animals were in strict accordance with guidelines of the Physiological Society of Japan and were approved by the Animal Research Committee of Kochi Medical School (Permit

*Corresponding author: Mikihiko Arikawa, PhD, Department of Cardiovascular Control, Kochi Medical School, Nankoku, Kochi 783-8505, Japan. Tel: +81-88-8802587; Fax: +81-88-880-2310; E-mail: marikawa@kochi-u.ac.jp

Received: June 14, 2014; Accepted: September 24, 2014; Published: September 26, 2014

Citation: Arikawa M, Kakinuma Y, Noguchi T, Sato T (2014) Donepezil, Therapeutic Acetylcholinesterase Inhibitor, Prevents the Progression of Ventricular Dysfunction by Promoting Myocardial Glucose Utilization in Rat Model of Chronic Heart Failure Following Myocardial Infarction. Cardiol Pharmacol 3: 121. doi:10.4172/23296607.1000121

Copyright: ( 2014 Arikawa M et al. This is an open-access article distributed under the terms of the Creative Commons Attribution License, which permits unrestricted use, distribution, and reproduction in any medium, provided the original author and source are credited. 
Citation: Arikawa M, Kakinuma Y, Noguchi T, Sato T (2014) Donepezil, Therapeutic Acetylcholinesterase Inhibitor, Prevents the Progression of Ventricular Dysfunction by Promoting Myocardial Glucose Utilization in Rat Model of Chronic Heart Failure Following Myocardial Infarction. Cardiol Pharmacol 3: 118. doi:10.4172/2329-6607.1000121

Number: D-00019). Male Wistar rats (aged 9-11 weeks and weighing 200-270 g) were purchased from Japan SLC (Hamamatsu, Japan) and were allowed free access to food and water or drug in a temperaturecontrolled room with a 12/12-hr light/dark cycle.

\section{Animal model and drug administration}

After induction of anesthesia with sodium pentobarbital $(50 \mathrm{mg} /$ $\mathrm{kg}$, intraperitoneally), rats were artificially ventilated (5\% carbon dioxide and $95 \%$ oxygen) using a volume-controlled Rodent Ventilator Model 683 (Harvard Apparatus, MA, USA) with a stroke volume of 3 $\mathrm{mL}$ and a respiratory rate of 60 strokes $/ \mathrm{min}$. A left-side thoracotomy was performed to expose the heart, and MI was induced by permanent occlusion of the left coronary anterior descending branch with a 7-0 silk suture (Akiyama MEDICAL MFG, Tokyo, Japan). Myocardial ischemia was confirmed by elevation of the ST segment on the electrocardiogram. After rats recovered from ventricular arrhythmias, which frequently occur in an ischemic heart, the thorax was closed with a 4-0 polyester suture (Bear Medic, Tokyo, Japan). In a sham group of rats, the same surgical procedures were performed except for the ligation of the coronary artery. During surgery, rats were placed on a warming pad to maintain the body temperature at approximately $37^{\circ} \mathrm{C}$. Infarcted rats were randomly divided into two groups: untreated and donepezil-treated (DPZ) groups. Donepezil (Eisai Co., Ltd., Tokyo, Japan) was dissolved in drinking water and administrated to rats for 8 weeks at a dosage of $5.0 \mathrm{mg} / \mathrm{kg} /$ day. Donepezil was administrated not only to sham-operated and infarcted rats but also to normal rats for 7 days at the same concentration to investigate the effect of donepezil on myocardial energy metabolism.

\section{Tail-cuff system and Langendorff-perfused heart preparation}

In sham-operated and infarcted rats, heart rate was measured noninvasively by a tail-cuff system with data analysis software (BP98A, Softron, Tokyo, Japan) at 8 weeks postoperatively. Thereafter, cardiac contractility was evaluated in isolated and Langendorffperfused hearts prepared under isovolumic conditions following our previously reported method with some modifications [16]. The details are described in the supplementary material. Left ventricular peak systolic pressure (LVPSP), left ventricular end-diastolic pressure (LVEDP), and left ventricular developed pressure (LVDP, an index of cardiac contractility defined as the difference between LVPSP and LVEDP) were measured at different left ventricular volumes. The left ventricular end-systolic elastance (Ees) was estimated from linear regression analysis of the pressure-volume curves at $100 \mathrm{mmHg}$ of LVPSP according to Sato et al. [17].

\section{Cardiomyocyte isolation and drug treatment}

Cardiomyocytes were harvested from 2-3-day-old neonatal rats by a collagenase/trypsin digestion protocol as described previously [18]. The details are described in the supplemental material. Isolated cardiomyocytes were seeded into 6-well or 12-well plates at a density of more than $1 \times 10^{6} \mathrm{cells} / \mathrm{cm}^{2}$ and incubated at $37^{\circ} \mathrm{C}$ for more than $24 \mathrm{hr}$. After medium was replaced with fresh conditioned Dulbecco's Modified Eagle Medium (DMEM, Wako Pure Chemical Industries, Lt., Osaka, Japan) with or without donepezil at a concentration of $10 \mu \mathrm{M}$, cells were further incubated at $37^{\circ} \mathrm{C}$ for $2-3$ days. The concentration of donepezil was determined by referring to Takada et al. who investigated the cell protective properties of donepezil [19]. mRNA or protein was obtained from the cultured cardiomyocytes for reverse transcription-polymerase chain reaction (RT-PCR) or Western blot analysis, respectively. Before harvesting, cellular beating was observed under a conventional inverted microscope (CKX41SF, Olympus Corporation, Tokyo, Japan) and expressed as beats per minute (bpm).

\section{Gene and protein expression analysis}

RT-PCR and Western blot analyse were performed according to the methods described previously [10,20]. The methods are detailed in the supplementary material.

\section{Glucose uptake assay}

To directly monitor glucose uptake in living cells, a fluorescently labeled deoxyglucose analog, 2-NBDG [2-(N-(7-nitrobenz-2-oxa-1,3diazol-4-yl)amino)-2-deoxyglucose, Life Technologies Japan Ltd., Tokyo, Japan] was used. Cardiomyocytes isolated from rat neonates were cultured on glass bottom dishes (Matsunami Glass Industry, Ltd., Tokyo, Japan) in the conditioned DMEM described above with or without donepezil $(10 \mu \mathrm{M})$ at $37^{\circ} \mathrm{C}$ for 3 days. Cells were washed twice with a phosphate-buffered saline (PBS) and then exposed to 2-NBDG $(100 \mu \mathrm{M})$ in the conditioned DMEM at $37^{\circ} \mathrm{C}$ for $10 \mathrm{~min}$. After cells were washed again with PBS, intracellular signals of 2-NBDG were observed and photographed with a confocal laser scanning microscope FV-300 (Olympus, Tokyo, Japan). Intensity values of the fluorescence signals of 2 -NBDG within randomly selected areas $\left(400 \mu \mathrm{m}^{2}\right)$ in captured digital images were quantitatively measured to evaluate the effect of donepezil on cellular glucose uptake ability.

\section{Statistical analysis}

Results are presented either as means \pm standard deviations (SDs) or a percentage compared with an untreated control sample. Nonparametric comparisons between two groups were performed with the Mann-Whitney U-test. Multiple comparisons among groups were performed with a nonparametric one-way analysis of variance using the Kruskal-Wallis test. A value of $P<0.05$ was considered statistically significant.

\section{Results}

\section{Effect of donepezil on cardiac contractility in rats following MI}

At 8 weeks after surgical induction of MI, LVPSP and LVEDP were measured isovolumically at different left ventricular volumes in Langendorff-perfused hearts of sham-operated (sham) and MI rats (untreated and DPZ) to evaluate the effect of donepezil on cardiac contractility. As shown in Figure 1a, the left ventricular volume at $100 \mathrm{mmHg}$ of LVPSP was significantly greater in the infarcted groups (untreated, $348 \pm 75 \mu \mathrm{L}, P<0.05$; DPZ, $332 \pm 58 \mu \mathrm{L}, P<0.05$ ) than in the sham group (sham, $55 \pm 8 \mu \mathrm{L}$, Table 1 ). Moreover, Ees was significantly lower in the infarcted groups (untreated, $0.24 \pm 0.06 \mathrm{mmHg} / \mu \mathrm{L}$, $P<0.05$; DPZ, $0.28 \pm 0.08 \mathrm{mmHg} / \mu \mathrm{L}, P<0.05)$ than in the sham group (sham, $0.92 \pm 0.11 \mathrm{mmHg} / \mu \mathrm{L}$, Table 1 ). These results indicate that cardiac dilatation and pump dysfunction progresses during the 8 weeks following MI. The DPZ group had a slightly higher LVPSP and lower LVEDP compared with the untreated group at more than $200 \mu \mathrm{L}$ of the left ventricular volume (Figure 1a), resulting in a significantly higher peak LVDP in the DPZ group than in the untreated group (untreated, $105 \pm 11 \mathrm{mmHg}$; DPZ, $125 \pm 11 \mathrm{mmHg}, P<0.05$, Figure $1 \mathrm{~b}$ and Table 1). Heart rate was comparable between the infarcted groups (untreated, $364 \pm 10$ bpm; DPZ, $346 \pm 22 \mathrm{bpm}, P>0.05$, Table 1). Moreover, the heart weight to body weight ratio was significantly decreased in the DPZ group compared with the untreated group (untreated, 4.52 $\pm 0.24 \mathrm{mg} / \mathrm{g}$; DPZ, $3.91 \pm 0.48 \mathrm{mg} / \mathrm{g}, P<0.05$, Table 1$)$. These results indicate that donepezil prevents the progression of postinfarct left 
Citation: Arikawa M, Kakinuma Y, Noguchi T, Sato T (2014) Donepezil, Therapeutic Acetylcholinesterase Inhibitor, Prevents the Progression of Ventricular Dysfunction by Promoting Myocardial Glucose Utilization in Rat Model of Chronic Heart Failure Following Myocardial Infarction. Cardiol Pharmacol 3: 118. doi:10.4172/2329-6607.1000121

ventricular dysfunction and sustains the cardiac contractile function independently of heart rate.

\section{Effect of donepezil on cardiac energy metabolism in rats following MI}

To understand the mechanism by which donepezil achieves cardioprotective effect in rats with $\mathrm{CHF}$, we investigated the left ventricular mRNA expression of genes related to cardiac energy metabolism, particularly genes involved in energy substrate uptake. The mRNA expression of CD36, which is involved in fatty acid uptake in the heart, and carnitine palmitoyltransferase I (CPT-I), which is a key regulatory enzyme in the fatty acid metabolic pathway, was significantly higher in the untreated group than in the sham group (CD36: untreated, $132 \pm 6 \%, P<0.01$; CPT-I: untreated, $114 \pm 9 \%$, $P<0.05$, Figures $2 \mathrm{a}, 2 \mathrm{~b})$. The DPZ group did not show increased expression of these genes (CD36: DPZ, $83 \pm 7 \%$; CPT-I: DPZ, $98 \pm$ $9 \%$, Figures $2 \mathrm{a}, 2 \mathrm{~b})$. On the contrary, the mRNA expression of GLUTs type 1 (GLUT1) and type 4 (GLUT4), which are membrane proteins that facilitate cellular glucose uptake, was significantly lower in the untreated group than in the sham group (GLUT1: untreated, $90 \pm$ 9\%, $P<0.05$ and GLUT4: untreated, $81 \pm 7 \%, P<0.05$, Figures $2 \mathrm{c}, 2 \mathrm{~d}$ ). Although GLUT1 expression was not significantly different between the infarcted groups (DPZ: $90 \pm 8 \%, P>0.05$ vs. untreated, Figure $2 \mathrm{c}$ ), GLUT4 expression in the DPZ group was significantly higher than that in the untreated group (DPZ: $104 \pm 9 \%, P<0.05$ vs. untreated, Figure $2 \mathrm{~d}$ ). These results indicate that donepezil alters the cardiac substrate preference from fatty acids to glucose for energy production in the chronic phase of our rat model of MI.

\section{Effect of donepezil on energy metabolism in cultured cardiomyocytes}

To investigate the direct effect of donepezil on myocardial energy metabolism, gene and protein expression analyses were conducted in vitro using isolated and cultured cardiomyocytes. RT-PCR analysis showed that the mRNA expression of genes related to fatty acid metabolism, CD36 and CPT-I, in DPZ cardiomyocytes was not significantly different from that in untreated cells (CD36: DPZ, $109 \pm$ $11 \%, P>0.05$; CPT-I: DPZ, $136 \pm 20 \%, P>0.05$ ), although a trend of increased expression was observed for both genes (Figures $3 a, 3 b$ ). On the other hand, the mRNA expression of GLUT1 and GLUT4 was significantly increased in DPZ cardiomyocytes (GLUT1: DPZ, 142 $\pm 20 \%, P<0.05$; GLUT4: DPZ, $160 \pm 20 \%, P<0.05)$ compared with untreated cells (Figures $3 c, 3 \mathrm{~d}$ ). The upregulating effect of donepezil on the expression of GLUTs was further confirmed at the protein level. Western blot analysis showed that, compared to that in the untreated cardiomyocytes, the protein expression of both GLUTs was significantly increased by donepezil treatment (GLUT1: DPZ, $133 \pm$ $11 \%, P<0.05$; GLUT4: DPZ, $180 \pm 8 \%, P<0.05$, Figures $4 \mathrm{a}, 4 \mathrm{~b})$.

Next, to directly monitor glucose uptake in living cells, the incorporation of 2-NBDG molecules was observed. Fluorescence microscopy and quantitative analysis of captured images showed that the fluorescence signal intensity of 2-NBDG in DPZ cells was significantly greater than that in untreated cells (DPZ, $185 \pm 60 \%$, $P<0.01$, Figures $5 \mathrm{a}, 5 \mathrm{~b})$. Pretreatment with $100 \mu \mathrm{M}$ fasentin, a glucose transporter inhibitor, blocked the upregulating effect of donepezil on $2-\mathrm{NBDG}$ uptake (fasentin/DPZ, $83 \pm 27 \% P<0.01$ vs. DPZ, Figures $5 \mathrm{a}, 5 \mathrm{~b})$. These results, together with the results described above, suggest that donepezil enhances glucose uptake by increasing GLUT expression

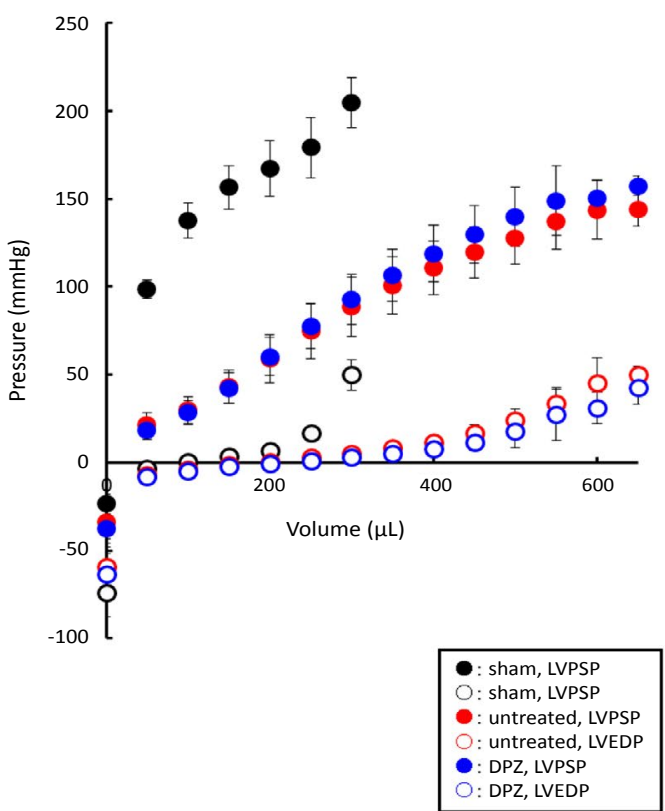

b

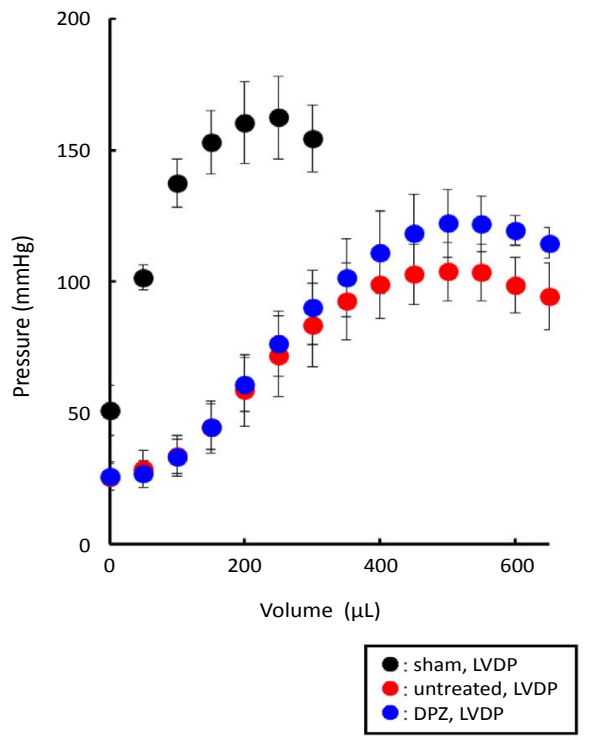

Figure 1: Pressure-volume relationships in sham-operated (sham) and MI rats [untreated and donepezil-treated (DPZ) groups] at 8 weeks postoperatively. (a) Left ventricular peak systolic pressure (LVPSP) -volume curves and left ventricular end-diastolic pressure (LVEDP) -volume curves. Black filled circles, sham LVPSP; black open circles, sham LVEDP; red filled circles, untreated LVPSP; red open circles, untreated LVEDP; blue filled circles, DPZ LVPSP; and blue open circles, DPZ LVEDP. The DPZ group had a slightly higher LVPSP and lower LVEDP compared with the untreated group at a left ventricular volume of more than $200 \mu L$. (b) Left ventricular developed pressure (LVDP) -volume curves. Black circles, sham LVDP; red circles, untreated LVDP; and blue circles, DPZ LVDP. The DPZ group had a significantly higher peak LVDP than the untreated group. Error bars represent standard deviations. 
Citation: Arikawa M, Kakinuma Y, Noguchi T, Sato T (2014) Donepezil, Therapeutic Acetylcholinesterase Inhibitor, Prevents the Progression of Ventricular Dysfunction by Promoting Myocardial Glucose Utilization in Rat Model of Chronic Heart Failure Following Myocardial Infarction. Cardiol Pharmacol 3: 118. doi:10.4172/2329-6607.1000121
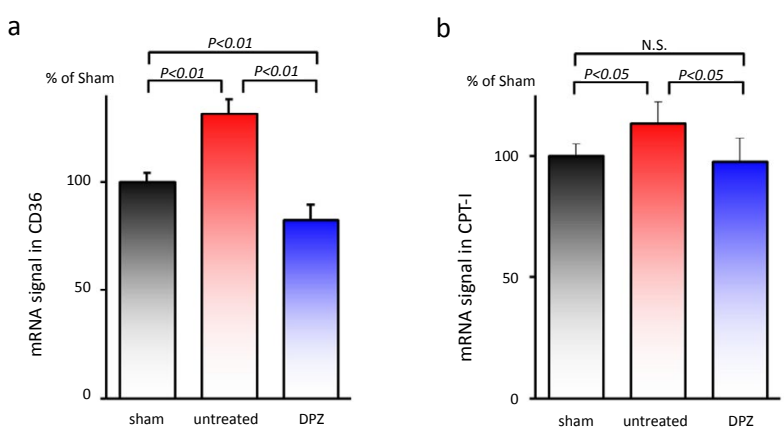

c
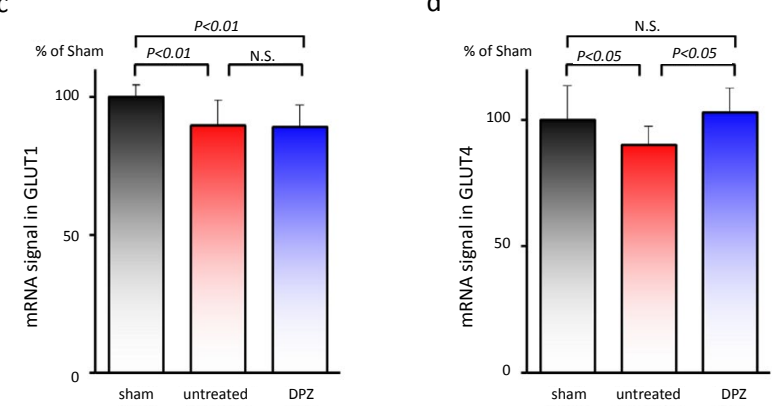

Figure 2: Left ventricular mRNA expression of genes related to myocardial energy metabolism in the sham-operated (sham) and MI rats [untreated and donepezil-treated (DPZ) groups] at 8 weeks postoperatively. (a) CD36, (b) CPT-I, (c) GLUT1, and (d) GLUT4. Black bars, sham; red bars, untreated; and blue bars, DPZ. The mRNA expression of CD36 and CPT-I was significantly higher in the untreated group than in the sham group and was significantly lower in the DPZ group than in the sham and untreated groups. On the contrary, the mRNA expression of GLUT1 and GLUT4 was significantly lower in the untreated group than in the sham group. Although there was no significant difference in GLUT1 expression between the infarcted groups, the mRNA expression of GLUT4 was significantly higher in the DPZ group than in the untreated group. Error bars represent standard deviations.

\begin{tabular}{|l|lll|}
\hline & sham & infarcted & \\
\hline & & untreated & DPZ \\
\hline $\mathrm{n}$ & 5 & 6 & 6 \\
\hline $\mathrm{HR}(\mathrm{bpm})$ & $338 \pm 21$ & $364 \pm 10$ & $346 \pm 22$ \\
\hline $\mathrm{LVV}(\mu \mathrm{L})$ & $55 \pm 8$ & $348 \pm 75 \#$ & $332 \pm 58 \#$ \\
\hline Ees $(\mathrm{mmHg} / \mu \mathrm{L})$ & $0.92 \pm 0.11$ & $0.24 \pm 0.06 \#$ & $0.28 \pm 0.08 \#$ \\
\hline $\begin{array}{l}\text { Peak LVDP } \\
(\mathrm{mmHg})\end{array}$ & $163 \pm 15$ & $105 \pm 11 \#$ & $125 \pm 11$ \#, \#\# \\
\hline HW/BW $(\mathrm{mg} / \mathrm{g})$ & $2.90 \pm 0.11$ & $4.52 \pm 0.24 \#$ & $3.91 \pm 0.48$ \#, \#\# \\
\hline
\end{tabular}

Values are means $\pm S D$. HR: heart rate; LVV: left ventricular volume at 100 $\mathrm{mmHg}$ of LVPSP; Ees: end systolic elastance at $100 \mathrm{mmHg}$ of LVPSP; LVDP: left ventricular developed pressure; HW/BW: heart weight to body weight ratio; DPZ: donepezil-treated group.

$\#, P<0.05$ versus sham group.

$\#$ \#, $P<0.05$ versus untreated group.

Table 1: Physiological characteristics in sham-operated and infarcted rats at 8 weeks following MI

in cardiomyocytes.

To further investigate the effect of donepezil on living cells, the rate of spontaneous beating was measured in cultured cardiomyocytes treated with or without donepezil. As shown in Figure 6, the beating rate of DPZ cells was significantly higher than that of untreated cells (untreated, $48 \pm 4 \mathrm{bpm}$; DPZ, $93 \pm 4 \mathrm{bpm}, P<0.01$ ). Fasentin treatment alone significantly lowered the beating rate (fasentin, $19 \pm 6 \mathrm{bpm}$, $P<0.01$ vs. untreated). The donepezil-induced increase in the beating rate was partially, but significantly, attenuated by fasentin treatment
(fasentin/DPZ, $66 \pm 12 \mathrm{bpm}, P<0.01$ vs. DPZ). The inhibitory effect of fasentin on the beating rate of untreated and DPZ cells was nullified after washing cells with fresh culture medium, which resulted in the full recovery of the rate [untreated (W), $49 \pm 7 \mathrm{bpm}$ and DPZ (W), 99 $\pm 12 \mathrm{bpm}, P<0.01]$. These findings indicate that donepezil increases the spontaneous beating rate of cardiomyocytes through the activation of glucose transporter function.

\section{Effect of donepezil on cardiac energy metabolism in normal rats}

The protein expression of GLUT1 and GLUT4 in cardiac tissues of normal rats treated with or without donepezil was evaluated by Western blot analysis. Compared with untreated rats, although the difference did not reach statistical significance for GLUT4 (DPZ, $119 \pm$ $37 \%, P>0.05$, Figure $7 \mathrm{~b}$ ), the cardiac protein expression of GLUT1 was significantly increased in DPZ rats (DPZ, $137 \pm 51 \%, P<0.05$, Figure $7 \mathrm{a})$.

\section{Discussion}

In the present study, we investigated the effect of donepezil on cardiac energy metabolism in an ischemic CHF model. Our results indicate that donepezil activates myocardial glucose metabolism by increasing the expression of GLUTs and accelerating glucose uptake, and thereby donepezil can confer resistance against ischemic conditions in cardiomyocytes and prevent the progression of ventricular dysfunction after $\mathrm{MI}$ in rats.

In this study, the chronic effect of donepezil treatment on cardiac contractility was evaluated in rats at 8 weeks after induction of MI. We used the isolated heart preparation method developed by Langendorff [21]. This vital technique is still widely used by a variety of cardiovascular researchers. Because the preparation is free of other organs, it allows the complete exclusion of peripheral influences on the isolated heart and the direct evaluation of heart function [22]. The analysis showed a slight elevation of LVPSP and a slight depression of LVEDP in the heart of DPZ rats, resulting in a significantly greater LVDP in the DPZ group than in the untreated group (Figure 1 and Table 1). Similar results were obtained in infarcted rats evaluated at 6 weeks after the induction of MI (data not shown), indicating that donepezil prevented the progression of postinfarct ventricular dysfunction during the treatment period.

Donepezil is the most frequently prescribed medication for the symptomatic treatment of patients with mild to moderate Alzheimer's disease worldwide. In this study, the dose of donepezil administrated to rats was $5.0 \mathrm{mg} / \mathrm{kg} / \mathrm{day}$, which was about 50 times higher than the therapeutic doses used in the clinical setting. However, although different from that used in humans, a daily dose of $5.0 \mathrm{mg} / \mathrm{kg}$ has been extensively used in animal studies without any side effects $[13,23]$. In a preliminary study, we confirmed that this dose of donepezil was the submaximal dose that did not cause side effects such as vomiting, diarrhea, anorexia, or growth retardation. Moreover, MI rats treated with a 10 times lower dose of donepezil $(0.5 \mathrm{mg} / \mathrm{kg} /$ day $)$ also showed preserved cardiac contractility comparably with those treated with the higher dose (data not shown), suggesting that the donepezil dose used in the present study $(5.0 \mathrm{mg} / \mathrm{kg} /$ day $)$ was not necessarily required for CHF treatment. Recently, the U. S. Food and Drug Administration (FDA) approved the dose of $23 \mathrm{mg}$ once daily in patients with moderate to severe Alzheimer's disease. This dose may cause slow heartbeat as a side effect [24]. However, we have reported that donepezil exhibits a cardioprotective effect in failing murine hearts in a heart rateindependent manner $[10,13]$. Also, in the present study, donepezil improved cardiac contractility without slowing heart rate (Table 1). These results suggest that the donepezil-induced cardioprotection is 
Citation: Arikawa M, Kakinuma Y, Noguchi T, Sato T (2014) Donepezil, Therapeutic Acetylcholinesterase Inhibitor, Prevents the Progression of Ventricular Dysfunction by Promoting Myocardial Glucose Utilization in Rat Model of Chronic Heart Failure Following Myocardial Infarction. Cardiol Pharmacol 3: 118. doi:10.4172/2329-6607.1000121

elicited not by its bradycardiac action but through its direct action on the failing hearts or damaged cardiomyocytes. Clinical trials have consistently shown the high efficacy, good safety, and favorable tolerability profiles of donepezil [25-27]. From the perspective of clinical use of donepezil for cardiovascular diseases, the effect of donepezil on mortality and prognosis in patients with heart failure seems to be very important in translational research. Our previous retrospective cohort investigation suggested a potential efficacy of donepezil in lowering cardiovascular mortality in patients with Alzheimer's disease [6]. Moreover, our recent prospective clinical study showed that no cardiovascular changes occurred during treatment with donepezil in patients with dementia [7]. These results, together with earlier clinical studies, suggest that donepezil can be a new potential candidate for a clinically useful drug applicable for the prevention and treatment of heart failure. A recently published clinical study also supports our proposed usage of acetylcholinesterase inhibitors including donepezil in the clinical setting, with an expected protective effect on overall cardiovascular mortality in patients with dementia $[28,29]$.

In the present study, we investigated the direct effect of donepezil on myocardial energy metabolism, particularly on the expression of genes involved in energy substrate uptake using isolated and cultured cardiomyocytes. We chose CD36 and CPT-I as the indices for fatty acid metabolism and GLUT1 and GLUT4 as the indices for glucose metabolism. The membrane protein CD36 plays an important role in long-chain fatty acid binding/transport from the extracellular compartment to the cytoplasm [30]. It has been demonstrated that mice null for CD36 exhibit reduced uptake and utilization of long-chain fatty acids in the heart [31], and that mice with CD36 overexpression in the muscle exhibit a decrease in serum fatty acids [32], indicating that CD36 is necessary for fatty acid uptake. CPT-I is a key enzyme involved in the regulation of fatty acid oxidation [33]. CPT-I is located on the outer mitochondrial membrane and converts long-chain fatty acyl-CoA to acylcarnitine. Because CPT-I is responsible for the first step in fatty acid oxidation in mitochondria, considerable attention has been paid to its functional features [34]. As shown in Figure 2, the cardiac mRNA expression of CD36 and CPT-I was higher in the untreated MI group than in the sham group, indicating that cardiomyocytes somehow attempted the uptake of fatty acids to efficiently produce energy in the failing heart. However, it seems to be a wasted effort due to myocardial ischemia resulting from poor oxygen supply. In vitro experiments using cultured cardiomyocytes showed that donepezil significantly increased the protein expression of GLUTs (Figure 4) and accelerated cellular glucose uptake (Figure 5). Moreover, oral administration of donepezil also increased the cardiac expression of GLUTs in normal and infarcted rats (Figures 2,7). Therefore, donepezil might have an ability to enhance cardiac glucose utilization regardless of the pathophysiological condition. In the DPZ group of MI rats, the mRNA expression of genes not related to fatty acid uptake but rather to glucose uptake was increased (Figure 2). When cells incorporate glucose, the transport of glucose across the plasma membrane occurs by facilitated diffusion through selective transport proteins of the GLUT family. In cardiomyocytes, the predominant glucose transport isoforms are GLUT1 and GLUT4 [35]. The GLUT1/GLUT4 ratio in rat hearts varies from 0.1 to 0.6 [36]. Because of its greater expression in cardiomyocytes, GLUT4 is responsible for the majority of glucose uptake. Therefore, the number of GLUT4 molecules present at the cell surface is the major determinant of glucose uptake into cardiomyocytes [37]. Among the donepezil-induced alterations in gene expression, the enhancement of glucose metabolism seemed to have a greater contributive effect for efficient energy production in CHF rather than the suppression of fatty acid metabolism. GLUT4-deficient mice exhibit cardiac hypertrophy and impaired cardiac contractile performance [38]. Moreover, transgenic mice with cardiac-specific overexpression of GLUT1 show attenuated development of cardiac failure when subjected to a chronic pressure overload [39]. These studies using genetically modified mice revealed that increasing myocardial glucose uptake and facilitating glycolysis without any alteration in fatty acid metabolism protected against cardiac dysfunction and prevented the progression to CHF. In cultured cardiomyocytes, as shown in Figure 3, donepezil increased mRNA expression of GLUTs without any changes in the expression of mRNA related to fatty acid incorporation, which may explain the cardioprotective effect of donepezil observed in cardiac tissue in DPZ a
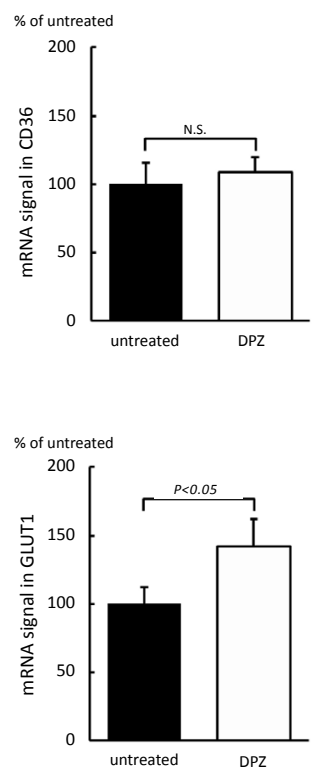

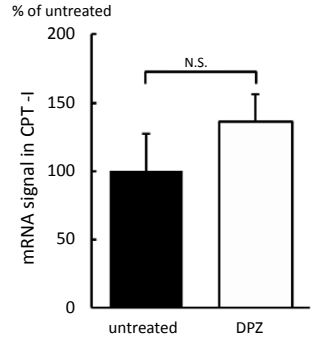

d

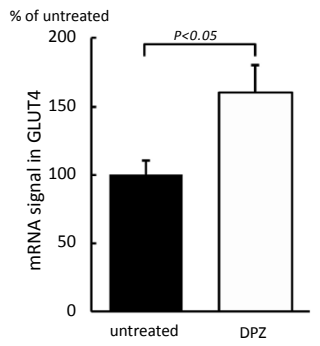

Figure 3: mRNA expression of genes related to myocardial energy metabolism in primary cultured rat cardiomyocytes with or without donepezil treatment. (a) CD36, (b) CPT-I, (c) GLUT1, and (d) GLUT4. Filled bars, untreated and open bars, donepezil-treated (DPZ) cells. Although the mRNA expression of CD36 and CPT-I was comparable between untreated and DPZ cardiomyocytes, the mRNA expression of GLUT1 and GLUT4 was significantly increased in the DPZ cardiomyocytes. Error bars represent standard deviations.

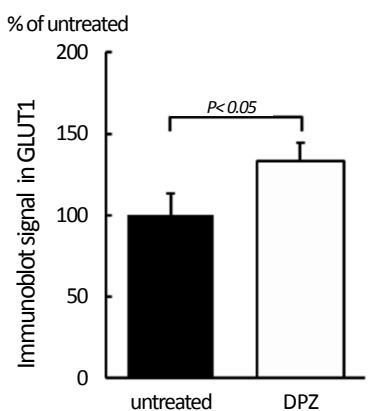

b

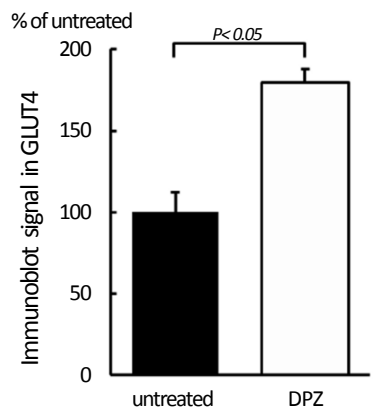

Figure 4: Protein expression of GLUT1 and GLUT4 in primary cultured rat cardiomyocytes with or without donepezil treatment. (a) GLUT1 and (b) GLUT4. Filled bars, untreated and open bars, donepezil-treated (DPZ) cells. Compared with the untreated cardiomyocytes, the protein expression of both glucose transporters (GLUTs) was significantly increased by donepezil treatment. Error bars represent standard deviations. 
Citation: Arikawa M, Kakinuma Y, Noguchi T, Sato T (2014) Donepezil, Therapeutic Acetylcholinesterase Inhibitor, Prevents the Progression of Ventricular Dysfunction by Promoting Myocardial Glucose Utilization in Rat Model of Chronic Heart Failure Following Myocardial Infarction. Cardiol Pharmacol 3: 118. doi:10.4172/2329-6607.1000121

rats following MI. It is still controversial whether other members of the GLUT family are expressed in the heart. Grover-McKay et al. showed that GLUT3 was present in adult and fetal human hearts [40]. On the contrary, Haber et al. reported that GLUT3 protein was expressed in testis and brain but not in the human heart [41]. To our knowledge, the cardiac expression of GLUT2 has not been investigated thus far.

It is usually accepted that cardiomyocytes preferentially utilize fatty acid oxidation to meet energy requirements when oxygen is abundant. Myocardial fatty acid oxidation is estimated to provide more than $70 \%$ of the cardiac energy demand under resting conditions $[42,43]$. However, a large amount of oxygen is required to utilize the aerobic pathway for energy production. Because glycolysis can occur when oxygen is depleted, it is reasonable to propose that facilitating the anaerobic pathway in cardiomyocytes is more efficient for producing energy under hypoxic conditions, and that cardiomyocytes incorporating glucose as an energy substrate of the glycolytic pathway acquire a level of resistance against ischemia. In this study, we showed that donepezil increased the cardiac expression of GLUTs, but the underlying mechanism remains uncertain. Based on the results of our previous study, together with others, the following mechanism is suspected. The pharmacological action of donepezil is based on an increase in the acetylcholine (ACh) level via inhibition of acetylcholinesterase activity. ACh has been reported to upregulate hypoxia-inducible factor (HIF)-1 alpha even in normoxic conditions [44]. HIF-1 alpha is well known as a master transcriptional factor that positively regulates the expression of glucose metabolism-related genes including GLUTs [45]. Therefore, it is conceivable that ACh and HIF-1 alpha play important roles in the donepezil-induced upregulation of GLUT expression. Our recent report that the newly developed heartspecific choline acetyltransferase (ChAT) transgenic mice show higher levels of ACh and HIF-1 alpha protein and also more augmented expression of cardiac GLUTs and increased cardiac glucose contents compared with those levels in the wild-type mouse heart [46] also further strengthens the involvement of ACh and HIF-1 alpha in the a
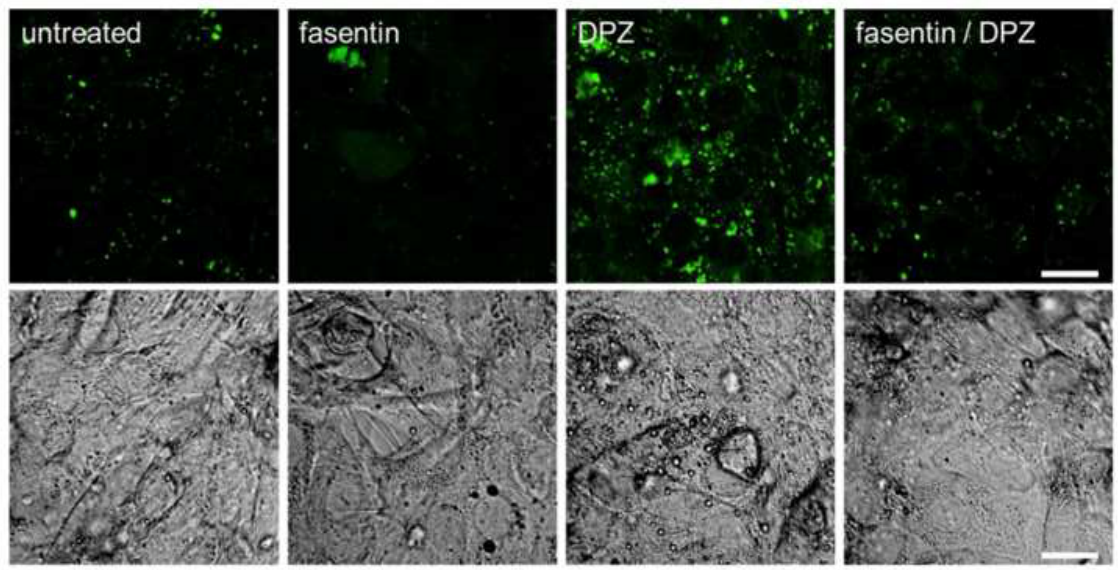

b

\section{$\%$ of untreated}

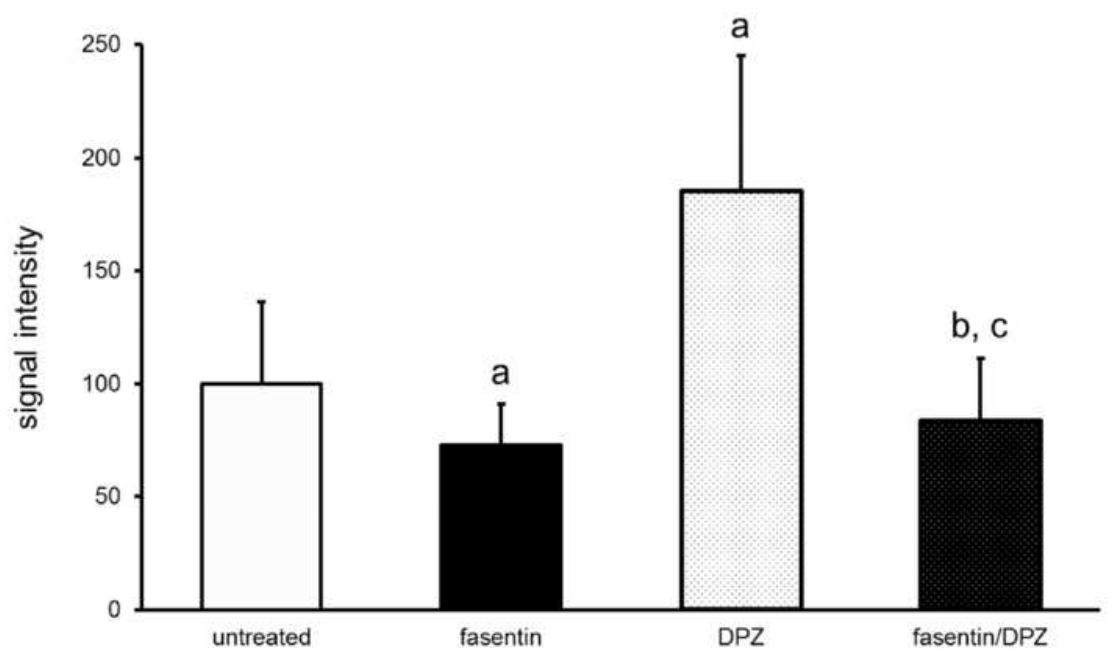

Figure 5: (a) Glucose uptake assay with a fluorescently labeled glucose analog 2-NBDG in primary cultured rat cardiomyocytes with or without donepezil treatment Upper and lower panels represent fluorescence micrographs showing signals of incorporated 2-NBDG in cardiomyocytes and differential interference contrast images corresponding to the fluorescent images, respectively. Scale bars represent $20 \mu \mathrm{m}$. (b) Quantitative analysis of fluorescence signal intensity of 2-NBDG in captured images presented in Figure 5a. The 2-NBDG fluorescence signal in DPZ cells (DPZ) was significantly greater than that in untreated cells (untreated). Pretreatment with $100 \mu \mathrm{M}$ fasentin, a glucose transporter inhibitor, blocked $2-N B D G$ uptake by DPZ cardiomyocytes (fasentin/DPZ). a, $P<0.01$ versus untreated. b, $P<0.05$ versus untreated. c, $P<0.01$ versus DPZ. Error bars represent standard deviations. 
Citation: Arikawa M, Kakinuma Y, Noguchi T, Sato T (2014) Donepezil, Therapeutic Acetylcholinesterase Inhibitor, Prevents the Progression of Ventricular Dysfunction by Promoting Myocardial Glucose Utilization in Rat Model of Chronic Heart Failure Following Myocardial Infarction. Cardiol Pharmacol 3: 118. doi:10.4172/2329-6607.1000121

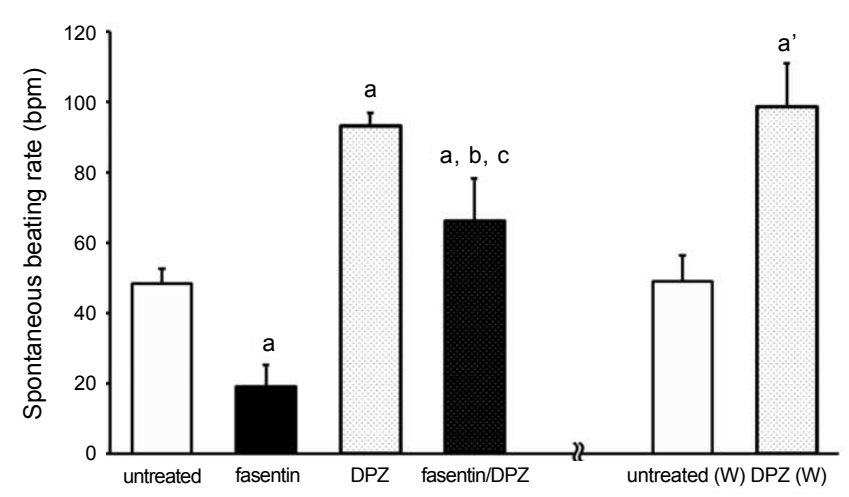

Figure 6: The effect of donepezil on the spontaneous beating rate of primary cultured rat cardiomyocytes with or without donepezil treatment. The beating rate of DPZ cells (DPZ) was significantly greater than that of untreated cells (untreated). Fasentin treatment significantly lowered the beating rate (fasentin). The donepezil-induced increase in the beating rate was partially attenuated by fasentin treatment (fasentin/DPZ). The inhibitory effect of fasentin on the beating rate of untreated and DPZ cells was fully recovered after cells were washed with fresh culture medium [untreated (W) and DPZ (W)]. a, $P<0.01$ versus untreated. a', $P<0.01$ versus untreated (W). b, $P<0.01$ versus fasentin. c, $P<0.01$ versus DPZ. Error bars represent standard deviations.

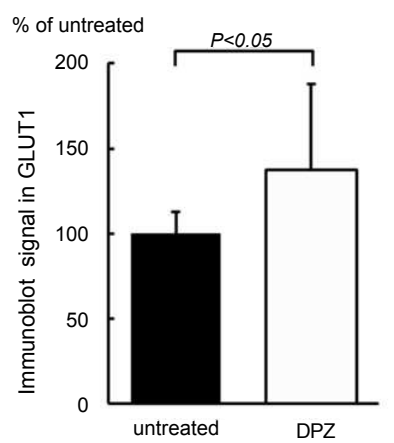

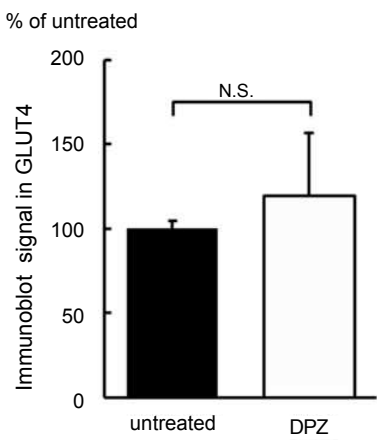

Figure 7: Left ventricular protein expression of GLUT1 (a) and GLUT4 (b) in untreated (untreated) and donepezil-treated (DPZ) rats. Although the difference in GLUT4 expression did not reach statistical significance (b), the cardiac protein expression of GLUT1 was significantly increased in the DPZ group compared with the untreated group (a). Error bars represent standard deviations.

mechanism of action of donepezil. Moreover, it has been reported that cardiomyocytes possess an ACh synthesis system that is activated by donepezil [11]. The non-neuronal cholinergic system might also be involved in efficient glucose metabolism in cardiomyocytes. Additionally, a number of experimental studies strongly suggest that donepezil directly affect cells via a mechanism that is independent of its pharmacological action of acetylcholinesterase inhibition [19,47-49]. Further investigation is needed to determine the molecular mechanism of the donepezil-induced increase in the efficiency of cardiac glucose utilization.

Primary cultured cardiomyocytes have been widely recognized as a valuable tool for pharmacological studies [50]. It can be observed under a microscope that cardiomyocytes start to beat spontaneously and synchronously after a few days in culture. In the present study, unexpectedly, the beating rate of cardiomyocytes treated with donepezil was approximately 2 -fold higher than that of untreated cells (Figure 6).
From the observed results, we hypothesize that cardiomyocytes treated with donepezil could more efficiently produce the energy required for greater cellular activity than the untreated cells via facilitation of a glycolytic metabolism that requires less oxygen. This hypothesis is also supported by our recently published study in which we found that ChAT-overexpressing cells have upregulated GLUT protein expression and glucose uptake as well as increased cellular ATP levels [51]. In the present study, we used fasentin, a glucose transporter inhibitor, to determine if donepezil actually affects glucose uptake and the spontaneous beating rate of cardiomyocytes through the function of GLUTs. Our results show that fasentin treatment fully blocks the donepezil-induced increase in glucose uptake (Figure 5) and partially attenuates the donepezil-induced increase in the beating rate of cardiomyocytes (Figure 6). Fasentin is known to be a GLUT1 inhibitor [52]. Using virtual docking studies, fasentin was found to inhibit glucose uptake by binding the intramembrane channel of GLUT1 [53]. Therefore, the selective inhibitory action of fasentin against GLUTs might account for the insufficient inhibition of the facilitative effect of donepezil on cardiomyocyte beating. Further investigation using an inhibitor specific for GLUT4 or all members of the GLUT family is needed to clarify the molecular mechanisms of the donepezil-induced increase in cardiac glucose metabolism. Another molecular approach involving a knockdown assay using short hairpin RNA or small interfering RNA specific for GLUT1 and/or GLUT4 seems to offer a method for definitively identifying which GLUT isoform plays the most important role in the transport of glucose in cardiomyocytes.

In conclusion, the present study showed for the first time that donepezil has the ability to facilitate cellular glucose utilization by increasing the expression of myocardial GLUTs, and through this action, donepezil prevented the progression of cardiac dysfunction and sustained the left ventricular contractility without affecting heart rate during the chronic phase of $\mathrm{MI}$ in rats. Therefore, donepezil represents a new potentially clinically useful drug candidate for CHF therapy.

\section{Acknowledgments}

The authors gratefully acknowledge Ms. Kayo Okazaki for her generous technical assistance throughout the study. We also thank Drs. Rajesh G Katare (University of Otago, New Zealand) and Motonori Ando (Okayama University, Japan) for their helpful discussion.

\section{Sources of Funding}

This work was supported by a Grant-in-Aid for Young Scientists from the Japan Society for the Promotion of Science (Grant Number 23790858). The funders had no role in the study design, data collection and analysis, decision to publish, or preparation of the manuscript.

\section{References}

1. Taegtmeyer H (2002) Switching metabolic genes to build a better heart Circulation 106: 2043-2045.

2. Taegtmeyer $\mathrm{H}$ (2004) Cardiac metabolism as a target for the treatment of heart failure. Circulation 110: 894-896.

3. Barger PM, Kelly DP (1999) Fatty acid utilization in the hypertrophied and failing heart: molecular regulatory mechanisms. Am J Med Sci 318: 36-42.

4. Allard MF,SchonekessBO, Henning SL, English DR, Lopaschuk GD (1994) Contribution of oxidative metabolism and glycolysis to ATP production in hypertrophied hearts. Am J Physiol 267: H742-750.

5. vanBilsen M, van Nieuwenhoven FA, van der Vusse GJ (2009) Metabolic remodelling of the failing heart: beneficial or detrimental? Cardiovasc Res 81: 420-428.

6. Sato K,Urbano R, Yu C, Yamasaki F, Sato T, et al. (2010) The effect of donepezil treatment on cardiovascular mortality. Clin Pharmacol Ther 88: 335-338.

7. Kubo T, Sato T, Noguchi T, Kitaoka H, Yamasaki F, Kamimura N, et al. (2012) Influences of donepezil on cardiovascular system-possible therapeutic 
Citation: Arikawa M, Kakinuma Y, Noguchi T, Sato T (2014) Donepezil, Therapeutic Acetylcholinesterase Inhibitor, Prevents the Progression of Ventricular Dysfunction by Promoting Myocardial Glucose Utilization in Rat Model of Chronic Heart Failure Following Myocardial Infarction. Cardiol Pharmacol 3: 118. doi:10.4172/2329-6607.1000121

Page 8 of 9

benefits for heart failure-donepezil cardiac test registry (DOCTER) study. J CardiovascPharmacol 60: 310-314.

8. Li M, Zheng C, Kawada T, Inagaki M, Shishido T, Sato T, et al. (2007) Restoration of vagal tone by donepezil markedly improves long-term survival in rats with incurably severe heart failure. FASEB J 21:13.

9. Okazaki Y, Zheng C, Li M, Sugimachi M (2010) Effect of the cholinesterase inhibitor donepezil on cardiac remodeling and autonomic balance in rats with heart failure. J Physiol Sci 60: 67-74.

10. Arikawa M,Kakinuma Y, Handa T, Yamasaki F, Sato T (2011) Donepezil, anti-Alzheimer's disease drug, prevents cardiac rupture during acute phase of myocardial infarction in mice. PLoS One 6: e20629.

11. Kakinuma Y, Akiyama T, Sato T (2009) Cholinoceptive and cholinergic properties of cardiomyocytes involving an amplification mechanism for vagal efferent effects in sparsely innervated ventricular myocardium. FEBS J 276: 5111-5125.

12. Kakinuma Y, Furihata M, Akiyama T, Arikawa M, Handa T, et al. (2010) Donepezil, an acetylcholinesterase inhibitor against Alzheimer's dementia, promotes angiogenesis in an ischemic hindlimb model. $\mathrm{J}$ Mol Cell Cardiol 48:680-93

13. Handa T,Katare RG, Kakinuma Y, Arikawa M, Ando M, et al. (2009) AntiAlzheimer's drug, donepezil, markedly improves long-term survival after chronic heart failure in mice. J Card Fail 15: 805-811.

14. Tsuno N (2009) Donepezil in the treatment of patients with Alzheimer's disease. Expert Rev Neurother 9: 591-598.

15. Doody RS, Cummings JL, Farlow MR (2012) Reviewing the role of donepezil in the treatment of Alzheimer's disease. Curr Alzheimer Res 9: 773-781.

16. Katare RG, Ando M, Kakinuma Y, Arikawa M, Handa T, et al. (2009) Vagal nerve stimulation prevents reperfusion injury through inhibition of opening of mitochondrial permeability transition pore independent of the bradycardiac effect. J ThoracCardiovascSurg 137: 223-231.

17. Sato T, Shishido T, Kawada T, Miyano H, Miyashita H, et al. (1998) ESPVR of in situ rat left ventricle shows contractility-dependent curvilinearity. Am J Physiol 274: H1429-1434.

18. Ando M,Katare RG, Kakinuma Y, Zhang D, Yamasaki F, et al. (2005) Efferent vagal nerve stimulation protects heart against ischemia-induced arrhythmias by preserving connexin43 protein. Circulation 112: 164-170.

19. Takada Y, Yonezawa A, Kume T, Katsuki H, Kaneko S, et al. (2003) Nicotinic acetylcholine receptor-mediated neuroprotection by donepezil agains glutamate neurotoxicity in rat cortical neurons. J PharmacolExp Ther 306: 772-777.

20. Noguchi T, Kakinuma Y, Arikawa M, Okazaki K, Hoshino E, et al. (2014) Donepezil can improve ischemic muscle atrophy by activating angiomyogenic properties of satellite cells. Circ J 78: 2317-2324.

21. Langendorff $O$ (1895) Untersuchungen am überlebendenSäugetierherzen. PflügersArchiv 61: 291-332.

22. Skrzypiec-Spring M, Grotthus B, Szelag A, Schulz R (2007) Isolated heart perfusion according to Langendorff---still viable in the new millennium. J Pharmacol Toxicol Methods 55: 113-126.

23. Inanaga K,Ichiki T, Miyazaki R, Takeda K, Hashimoto T, et al. (2010) Acetylcholinesterase inhibitors attenuate atherogenesis in apolipoprotein E-knockout mice. Atherosclerosis 213: 52-58.

24. ARICEPT donepezil hydrochloride tablets. [http://www.aricept.com/]

25. Rogers SL,Doody RS, Mohs RC, Friedhoff LT (1998) Donepezil improves cognition and global function in Alzheimer disease: a 15-week, double-blind, placebo-controlled study. Donepezil Study Group. Arch Intern Med 158 1021-1031.

26. Doody RS, Corey-Bloom J, Zhang R, Li H, leni J, et al. (2008) Safety and tolerability of donepezil at doses up to $20 \mathrm{mg} /$ day: results from a pilot study in patients with Alzheimer's disease. Drugs Aging 25: 163-174.

27. Farlow M, Veloso F, Moline M, Yardley J, Brand-Schieber E, et al. (2011) Safety and tolerability of donepezil $23 \mathrm{mg}$ in moderate to severe Alzheimer's disease. BMC Neurol 11: 57

28. Nordstrom P, Religa D, Wimo A, Winblad B, Eriksdotter M (2013) The use of cholinesterase inhibitors and the risk of myocardial infarction and death: a nationwide cohort study in subjects with Alzheimer's disease. Eur Heart J 34 2585-2591.

29. Torjesen I (2013) Study links drugs for Alzheimer's disease with reduced risk of heart attack and death. BMJ 346: f3669.

30. Aitman TJ, Glazier AM, Wallace CA, Cooper LD, Norsworthy PJ, et al. (1999) Identification of Cd36 (Fat) as an insulin-resistance gene causing defective fatty acid and glucose metabolism in hypertensive rats. Nat Genet 21: 76-83.

31. Coburn CT, Knapp FF Jr, Febbraio M, Beets AL, Silverstein RL, et al. (2000) Defective uptake and utilization of long chain fatty acids in muscle and adipose tissues of CD36 knockout mice. J Biol Chem 275: 32523-32529.

32. Ibrahimi A, Bonen A, Blinn WD, Hajri T, Li X, et al. (1999) Muscle-specific overexpression of FAT/CD36 enhances fatty acid oxidation by contracting muscle, reduces plasma triglycerides and fatty acids, and increases plasma glucose and insulin. J Biol Chem 274:26761-26766.

33. Wang D, Harrison W, Buja LM, Elder FF, McMillin JB (1998) Genomic DNA sequence, promoter expression, and chromosomal mapping of rat muscle carnitinepalmitoyltransferase I. Genomics 48: 314-323.

34. Yamazaki N (2004) Identification of muscle-type carnitinepalmitoyltransferase I and characterization of its atypical gene structure. Biol Pharm Bull 27: 17071716.

35. Abel ED (2004) Glucose transport in the heart. Front Biosci 9: 201-215.

36. Kraegen EW, Sowden JA, Halstead MB, Clark PW, Rodnick KJ, et al. (1993) Glucose transporters and in vivo glucose uptake in skeletal and cardiac muscle: fasting, insulin stimulation and immunoisolation studies of GLUT1 and GLUT4 Biochem J 295 : 287-293.

37. Montessuit C, Lerch R (2013) Regulation and dysregulation of glucose transport in cardiomyocytes. Biochim Biophys Acta 1833: 848-856.

38. Huggins CE, Domenighetti AA, Ritchie ME, Khalil N, Favaloro JM, et al. (2008) Functional and metabolic remodelling in GLUT4-deficient hearts confers hyperresponsiveness to substrate intervention. J Mol Cell Cardiol 44: 270-280.

39. Liao R, Jain M, Cui L, D’Agostino J, Aiello F, et al. (2002) Cardiac-specific overexpression of GLUT1 prevents the development of heart failure attributable to pressure overload in mice. Circulation 106: 2125-2131.

40. Grover-McKay M, Walsh SA, Thompson SA (1999) Glucose transporter 3 (GLUT3) protein is present in human myocardium. Biochim Biophys Acta 1416: $145-154$

41. Haber RS, Weinstein SP, O'Boyle E, Morgello S (1993) Tissue distribution of the human GLUT3 glucose transporter. Endocrinology 132: 2538-2543.

42. Stanley WC,Recchia FA, Lopaschuk GD (2005) Myocardial substrate metabolism in the normal and failing heart. Physiol Rev 85: 1093-1129.

43. Lionetti V, Stanley WC, Recchia FA (2011) Modulating fatty acid oxidation in heart failure. Cardiovasc Res 90: 202-209.

44. Kakinuma Y, Ando M, Kuwabara M, Katare RG, Okudela K, et al. (2005) Acetylcholine from vagal stimulation protects cardiomyocytes against ischemia and hypoxia involving additive non-hypoxic induction of HIF-1alpha. FEBS Lett 579: 2111-2118.

45. Loor G, Schumacker PT (2008) Role of hypoxia-inducible factor in cell survival during myocardial ischemia-reperfusion. Cell Death Differ 15: 686-690.

46. Kakinuma Y, Tsuda M, Okazaki K, Akiyama T, Arikawa M, et al. (2013) Heartspecific overexpression of choline acetyltransferase gene protects murine heart against ischemia through hypoxia-inducible factor-1alpha-related defense mechanisms. J Am Heart Assoc 2:e004887.

47. Takada-Takatori Y,Kume T, Ohgi Y, Izumi Y, Niidome T, et al. (2008) Mechanism of neuroprotection by donepezil pretreatment in rat cortical neurons chronically treated with donepezil. J Neurosci Res 86: 3575-3583.

48. Akasofu S, Kimura M, Kosasa T, Sawada K, Ogura H (2008) Study of neuroprotection of donepezil, a therapy for Alzheimer's disease. Chem Biol Interact 175: 222-226.

49. Akaike A, Takada-Takatori $Y$, Kume T, Izumi $Y$ (2010) Mechanisms of neuroprotective effects of nicotine and acetylcholinesterase inhibitors: role of alpha4 and alpha7 receptors in neuroprotection. J Mol Neurosci 40: 211-216.

50. Fu J, Gao J, Pi R, Liu P (2005) An optimized protocol for culture of cardiomyocyte from neonatal rat. Cytotechnology 49:109-116. 
Citation: Arikawa M, Kakinuma Y, Noguchi T, Sato T (2014) Donepezil, Therapeutic Acetylcholinesterase Inhibitor, Prevents the Progression of Ventricular Dysfunction by Promoting Myocardial Glucose Utilization in Rat Model of Chronic Heart Failure Following Myocardial Infarction. Cardiol Pharmacol 3: 118. doi:10.4172/2329-6607.1000121

Page 9 of 9

51. Oikawa S,Iketani M, Kakinuma Y (2014) A non-neuronal cholinergic system regulates cellular ATP levels to maintain cell viability. Cell Physiol Biochem 34: 781-789.

52. Andrisse S, Koehler RM, Chen JE, Patel GD, Vallurupalli VR, et al. (2014) Role of GLUT1 in regulation of reactive oxygen species. Redox Biol 2: 764-771.

53. Wood TE, Dalili S, Simpson CD, Hurren R, Mao X, et al. (2008) A novel inhibitor of glucose uptake sensitizes cells to FAS-induced cell death. Mol Cancer Ther 7: 3546-3555. 